\title{
VARIATION WITH PRESSURE OF THE RESIDUAL IONIZATION DUE TO THE PENETRATING - RADIATION
}

\author{
BY \\ K. MELVINA DOWNEY
}

\section{A THESIS}

PRESENTED to the FACULTy of THE Graduate SCHOOL of Uniyersity of MinNesota in Partial. FULFILLMENT of tHe REQUikements foR THE DEGREe OF DOCTOR OF PHILOSOPHY

THE NEW TRA PRES OF

LACNSTER, PA:

xga0 


\section{VARIATION WITH PRESSURE OF THE RESIDUAL IONIZATION DUE TO THE PENETRATING RADIATION}

BY

K. MELVINA DOWNEY

\section{A THESIS}

Presented to the Faculty of the Graduate School of University of Minnesota in Partial Fulfillment of the Requirements for the Degree of Doctor of Philosophy 
I Reprinted from the Physical Rzvizw, N.S., Vol. XVI. No. 5, November, 1920.]

\title{
VARIATION WITH PRESSURE OF THE RESIDUAL IONIZA- TION DUE TO THE PENETRATING RADIATION.
}

\author{
By K. Melvina Downey.
}

SYNOPSIS.

Residual Ionization in Closed Vessels: Experimental Melhod.-In order to throw light upon the type of radiation responsible for the residual ionization in closed vessels, the author has measured the ionization in a sphere one foot in diameler for pressures ranging up to 21.5 atmospheres. Any corpuscular radiation not absorbed in the ionization sphere has a penetration at one atmosphere equal to or greater than the product of the dimension of the sphere and the number of atmospheres pressure. A special device was used to compensate for any fuctuations of the battery furnishing the high potential required for saturation. This consisted of two ionization spheres of the same electrical capacity which were connected to the opposite ends of a ten megohm resistance, the mid-point of which was earthed. Connections from the battery were made to the ends of the ten megohms. The final experiments were performed over water to eliminate any effect due to radio-active substances in the soil.

Nature of Ionization Curve.-The shape of the ionization-pressure curves gives information as to the process of ionization. A discussion of such curves obtained by other observers has been given in connection with those of the author. The curves obtained in the present experiment give a linear relation. This indicates that the ionization is not due to a soft radiation from the walls of the sphere, but that it is due either to the direct action of a penetrating radiation without any effect produced by secondary $\beta$ rays from the gas, or to a secondary, corpuscular radiation from the walls of the vessel. If the latter is responsible for the main portion of the ionization, the experiments indicate that it has a penetration of at least six and one half meters in air at atmospheric pressure.

Diurnal Variation.-A few curves obtained during the preliminary observations have been given. Provided that the sky is not cloudy, these indicate the existence of a diurnal variation.

Ionization in Air Due to Gamma Rays of Radium.-The ionization in a sphere one foot in diameter has been measured for pressures ranging up to 40 atmospheres. From four to twenty atmospheres a linear relation existed. The change in slope at twenty atmospheres to a value about six tenths of that at lower pressures may be attributed to some of the $\beta$ rays having completed their paths. At the high pressures there is a suggestion of an effect due to the secondary radiation from the air.

\section{INTRODUCTION.}

I F air or any other gas is enclosed in a metallic vessel, experiments show that ions are produced in the gas at a constant rate even in the absence of any obviously apparent ionizing agent. The number of pairs of ions produced per c.c. per second varies slightly with the material of the vessel but over land is of the general order of magnitude of 10 . 
A part of this ionization is certainly due to the radiation from radioactive materials in the soil and the radio-active emanation in the air. That this source accounts for only a portion of the ionization is verified by the observations over the great oceans. Although the amount of radio-active material in these oceans and in the air over them is insignificant, yet values of the order of 4 pairs of ions per c.c. per second are obtained in closed vessels over these oceans. Thus, Simpson and Wright obtained values ranging from 4 to 6 ions per c.c. per second over the Atlantic and Indian oceans; $;^{1}$ and, on the fourth cruise of the yacht Carnegie $e^{2}$ an average value of 3.8 ions was obtained over the Pacific and sub-antarctic oceans. Again, when the ionization vessel is shielded on all sides by water sufficient in thickness to absorb the known radiation, there is still a residual ionization as was first shown by the experiments of Rutherford and Cooke. ${ }^{3}$

There are two views prevalent as to the origin of this residual ionization in gases. One attributes it to impurities in the walls of the vessel, and the other to a penetrating radiation having its origin outside the vessel. Much work has been done on this subject, particularly by McLennan and his students. An experiment of special interest is the one in which McLennan used an ionization 'chamber of ice. When this was placed over Lake Ontario, the ionization value obtained was only 2.6 ions per c.c. per second. McLennan seems to favor the view that even this residual ionization was due to radio-active impurities in the ice, but no test for the radioactivity of this ice-receiver appears to have been made.

Perhaps the most convincing evidence in favor of a true penetrating radiation in the atmosphere and against any explanation founded on the assumption of radio-active contamination is given by the balloon observations of Kleinschmidt and of Kohlhörster. The latter found, for example, that at an altitude of 9,300 meters, the value obtained for the residual ionization was 80.4 ions per c.c. per second. ${ }^{4}$ The value at the surface of the earth would be only about 4 ions per c.c. per second if we neglect the contribution due to the gamma rays from the radio-active material in the soil, which contribution would be quite negligible at an altitude of even 2,000 meters. Kohlhörster's results thus suggest a non-terrestrial radiation, increasing in intensity with altitude.

1 Roy. Soc. Proc., A, Vol. 85, pp. 196-198, 1911.

${ }^{2}$ Results of Atmospheric Electric Observations made aboard the Galilee (1907-1908) and the Carnegie (1909-1916), reprinted from Publication No. I75 (Vol. 3) of the Carnegie Inst. of Washington.

${ }^{2}$ Phys. Review, Vol. 16, p. $183,1903$.

4 Deutsch. Phys. Gesell. Verb., 16, p. $719,1904$. 
In the present work, the primary object was to measure the natural ionization in a closed vessel for pressures ranging up to 20 atmospheres, and to eliminate, as far as possible, the ionization resulting from radioactive materials in the soil by performing experiments over a body of water of sufficient thickness to cut off such radiation. The effects of pressure would then give information as to the nature of the agency responsible for the ionization. If the radiation is of an easily absorbable nature, the ionization will reach a saturation value at a comparatively low pressure; while for a range of pressures extending to even higher values the ionization would continue to increase in the case of the penetrating radiation. That is, the more penetrating the radiation, the higher the pressure for which a saturation value will be obtained. A more detailed discussion of the ionization-pressure relations, in so far as they are influenced by the properties of the radiation producing the ionization, will be given later. Several investigators have examined the variation of residual ionization for pressures below one atmosphere, ${ }^{1}$ while Wilson, ${ }^{2}$ and McLennan and Burton ${ }^{3}$ have made measurements for pressures above one atmosphere. These results will be discussed in connection with the distinctive features of the writer's experiments. It may be noted here that the pressure giving a saturation value of the ionization in any experiment depends upon the linear dimension of the ionization chamber, so that as much information may be given at a low pressure by a vessel of large dimensions as is given at a high pressure by a vessel of correspondingly smaller dimensions. In the course of the work it was desirable to observe also the variation of ionization with pressure when the ionization was caused by the rays from a sealed tube of radium bromide. These experiments, which were carried out up to 40 atmospheres, will be described in connection with the main experi-
ments.

\section{Description of Apparatus and Method.}

The form of apparatus used was suggested by Professor W. F. G. Swann. The general arrangement of the apparatus will be apparent from the diagram (Fig. I).

The ionization chambers $A$ and $B$ are two cast steel spheres each having an internal diameter of one foot and walls one inch in thickness. The central rod electrode of each sphere is joined to a common wire which in turn is connected to the fiber of a string electroscope of the ${ }^{1}$ For example: J. Patterson. Phil. Mag., Ser. 6, Vol. 6. p. 231, 1903, also Kingdom, Phil.
Mag., Vol. 32, p. 396, 1916.

2W. Wilson, Phil. Mag., Ser. 6, Vol. 17, p. $216,1909$.

'Phys, Review, Vol. 16, p. 184, 1903. 
Lutz-Edelmann type. ${ }^{1}$ The spheres are connected to the opposite ends of a ten megohm resistance. The mid-point of this resistance is connected to the earth terminal as are also the guard rings $G G$, the shielding tube $C$ and the electroscope case. Connections are made at the end terminals of the ten megohm resistance to the source of high potential

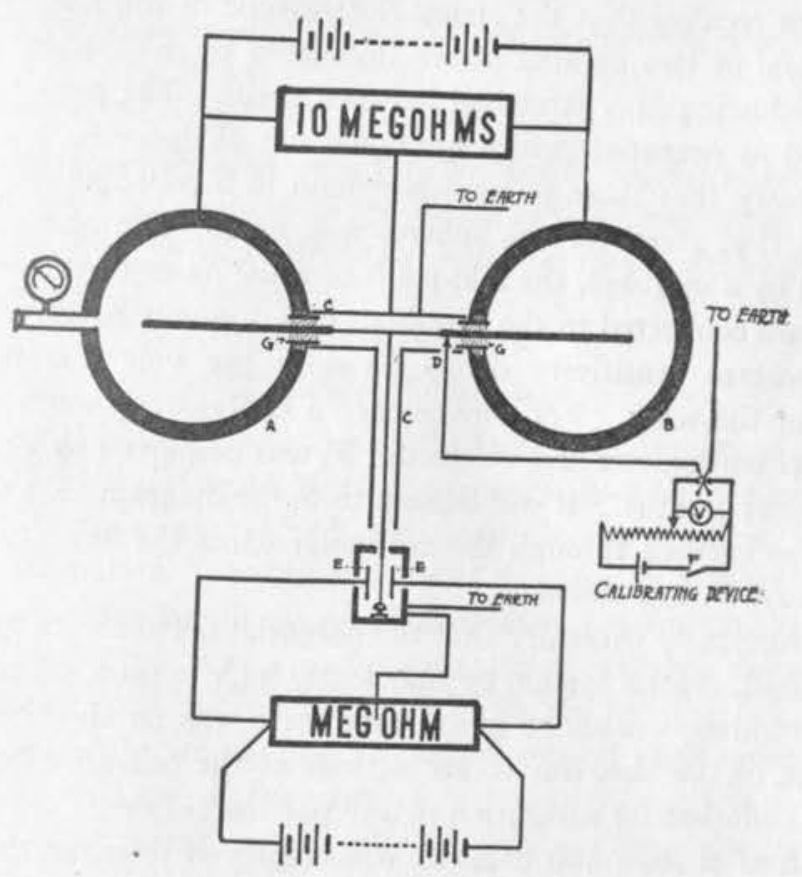

. Fig. 1.

(700 volts for the residual ionization.

I500 volts for the ionization due to rays from small sample of $\mathrm{Ra} \mathrm{Br}$.)

The use of the two vessels in combination with the ten megohm resistance possesses the advantage of compensating for any effects due to fluctuations of the batteries. These effects might otherwise, be serious on account of the high potentials necessary for saturation. On the other hand, by the use of two spheres with the ten megohm resistance, the midpoint of which is earthed, any alteration of the potential of the battery results in equal and opposite alterations of the potentials of the two ends of the resistance. Then the potentials of the two spheres are altered by equal and opposite amounts and the inductive actions of the two spheres on the central insulated system annul each other. The effect is the same as if the battery were constant. It is also apparent

${ }^{1}$ Lutz, Physikalische Zeitschrift, Vol. 13. p. 954, 1912. 
that there will be compensation as regards the residual ionization at atmospheric pressure if this is the same for the two spheres; consequently the rate of deflection of the electroscope fiber is determined by the excess ionization resulting from the increase of pressure in the sphere $A$ above the atmospheric pressure maintained in sphere $B$.

It will be recalled that the string electroscope of the Lutz-Edelmann type consists of two parallel plates (indicated by EE in the diagram) with a conducting fiber stretched between them. The plates are to be maintained at potentials which are equal but of opposite signs. Since it is necessary that these potentials remain in a constant ratio, a plan similar to that given for the spheres was used. The plates, $E E$, are connected by a megohm, the mid-point of which is earthed and the ends of which are connected to the terminals of a Ioo-volt battery. A practically constant sensitivity of Io divisions per volt was maintained throughout the work. For convenience a calibrating device consisting of a cell, potentiometer and voltmeter, $V$, was employed as a permanent part of the apparatus. It will be seen from the diagram that the central system was earthed through the voltmeter when the key, $F$, was open and the key, $D$, was closed.

It was obviously necessary that the potential between the spheres and the insulated, central system be sufficiently high to secure "saturation." Tests were always made to insure that there was no additional rate of movement of the fiber due to an increase of the potential beyond that assumed sufficient for saturation in any particular case.

A mixture of resin and beeswax was employed to- make the vessel $A$ airtight at high pressures. The bolts were tightened while the spheres were sufficiently hot to retain the mixture in a liquid form; and by this means it was possible to make the vessel stand a pressure of 65 atmospheres without appreciable leakage.

The pressure in $A$ was raised by connecting a valve at its end to a cylinder which had been filled with compressed air from a liquid air machine, and the pressure was recorded by a "Bourdon" gauge. The air was thoroughly dried by phosphorous pentoxide placed inside the sphere.

For the portion of the observations where a radio-active substance was required, a sample of radium bromide of the order of two milligrams was used. This was enclosed in a lead case, the walls of which were $I_{2}^{\frac{1}{2}}$ $\mathrm{cm}$. in thickness. The lead case containing the $\mathrm{Ra} \mathrm{Br}$. was placed at a distance of eight feet from the spheres during the observations in which it was used.

Method of Calculation.-The excess rate of production of ions per c.c. 
as a result of the increase in pressure in $A$ above atmospheric pressure is determined as follows:

Let $C=$ capacity of system,

$\Delta V=$ gain in potential of the fiber in e-s units,

$e=$ electronic charge $=4.8 \times \mathrm{Io}^{-10}$ e.s. units,

$v=$ vol. of ionization chamber in c.c.,

$q=$ number of pairs of ions produced per c.c., per sec.

We then have $q=\frac{C \Delta V}{v e t}$,

where $t$ is the number of seconds for the fiber to acquire a potenital $\Delta V$.

The order of magnitude of the emanation effects from the table, equipment and platform would be so small that they could be disregarded in the calculations.

\section{Measurements.}

Subsidiary Experiments.

Laboratory Conditions.- In view of the fact that a number of the experiments had to be performed in the laboratory, and since a great many radio-active measurements had been made in the building, it seemed necessary to make a thorough survey of the various laboratories so as to detect any effect due to any possible radio-active contamination of the walls. Furthermore, it was necessary to know whether there was any influence due to the radium known to be in the building. There were $2 \mathrm{mg}$. of radium in solution on the top floor and there were $3 \mathrm{mg}$. of shielded radium kept in the vault on the first floor. The laboratory, where the experiments were finally carried out, was situated in the basement.

The ionization chamber for these measurements was a cylindrical vessel having a volume of 6.8 liters. The central rod which was insulated from the vessel was connected to the string electrometer, and a connection was made to the calibrating device in a manner similar to that already given for the main apparatus. The potential (100 volts) which was applied to the vessel caused the ions of the same sign to go to the central electrode; when the connection to earth was released, the fiber would deflect. The time of the deflection of the fiber for a fixed number of divisions was recorded. The sensitivity of the electrometer was maintained at ro divisions per volt.

The following are representative of the observations:

Electrical Laboratory (top floor), Dec. 26, I918, values for $q$ : 10.4, $8.75,9.8,9.0,9.7$, 10.0 (Mean 9.6).

Room I (Basement), Dec. 27 , values for $q: 9.8,9.4,9.2,8.2,8.7$, 10.0

(Mean 9.2). 
Room I (Basement), Jan. II, $q$ : I0.8, I1.7, I2.6, I2.6 (Mean I1.9). Room II (Basement), Jan. II, $q$ : I0, 12.7, I0.8, II.7 (Mean I1.3).

In view of the fact that the effects due to passing clouds and to the other conditions determining $q$ are such variable quantities at different times, it is evident that there is no systematic difference between the values obtained for $q$ in Room I. and Room II. To make a still more conclusive test, the radium in the vault was removed to the vault in the library building. The values obtained were these:

Jan. 23-Before the removal of the Ra.-I2.2, II.9, II.9, II.5, II.9, I2.6 (Mean 12.0).

Jan. 23-After the removal of the Ra.-I2.4, I2.8, I2.4, 12.3, I2.2, 12.9 (Mean 12.5).

Jan. 24-Before the return of the Ra. to the Physics Building-(Io:30 to I I :30 A. M.) - I3 3.8, I2.7, I2.4, I3 12.8, I2.8 (Mean 12.9).

Jan. 24-After the return of the Ra.-(I2.15 to I :OO P. M.)-I3.0, I3.7,

12.3, 12.5, 13.2, 13.8 (Mean 13.1).

There was apparently no effect due to the presence of this radium in the vault. This was also verified by experiments of an entirely different nature which were performed by Miss Herrick (a graduate student in this department). ${ }^{1}$

Diurnal Variation.-During the course of the preceding observations, a decided variation in the ionization values was noticed at times. For quite a number of consecutive days in January, higher values were obtained in the evening than in the mid-part of the forenoon. Sometimes the difference was as much as 20 per cent.

In the interval from January 24 to February 6 , several series of observations were taken, each series extending over a period of 24 hours. When the atmosphere was exceedingly clear, a maximum was obtained in the morning ( 7 to 9 A.M.) and a more decided maximum from $6: 30$ to $8: 30$ P.M. with the values rather high during the first part of the night. When the sky was cloudy, no very obvious diurnal variation was obtained. The curves obtained January 26, February I and February 4 are given in Fig. 2. January 26 was an exceptionally clear day and there was no appreciable change of the barometer. February I was mostly cloudy. The obesrvations from February 3 to February 5 were begun just as a snow-fall, which had continued for some time, was ceasing. During a part of the observations on February 4 the sky was cloudy.

It would be desirable to continue these observations over a long period of time to test for the existence of the diurnal variation, particularly as there is not a unanimous agreement among investigators as to the

'Master's Thesis, University of Minnesota, rig. 
existence of the diurnal variation. The results obtained are in general agreement with the few observations recorded by those who have found a diurnal variation.

Saturation Voltage.-For the penetrating radiation the highest pressure used was not over 22 atmospheres. Tests for the saturation voltage

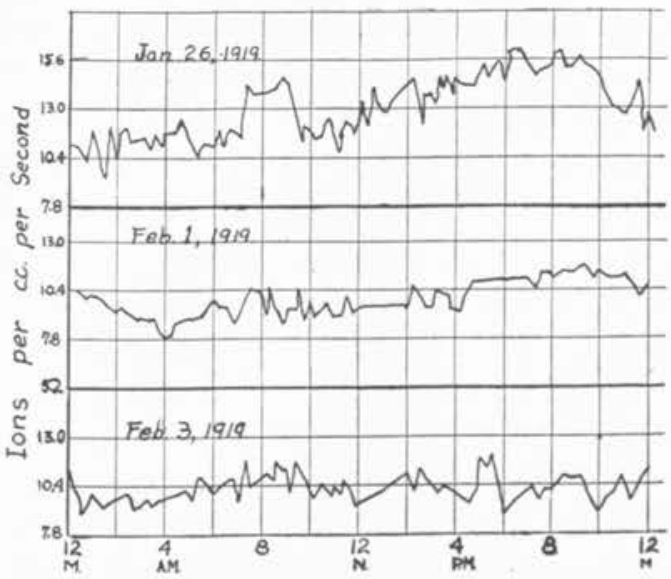

Fig. 2.

at this pressure were made. The potential of the end-terminals of the ten megohm resistance, and the corresponding values of the ionization current are given in Table I.

TABLE I.

Pressure, 22 Atmospheres

\begin{tabular}{l|c|c}
\hline Potential. & $\begin{array}{c}\text { Ionization } \\
\text { in Arbitrary } \\
\text { Units. }\end{array}$ \\
\hline 136 volts & 8.28 \\
272 & " & 8.37 \\
408 & " & 8.35 \\
544 & " & 8.33 \\
680 & " & 8.39 \\
\hline
\end{tabular}

TABLE II. Pressure, 20 Atmospheres.

\begin{tabular}{|c|c|}
\hline Potential. & $\begin{array}{l}\text { Ionization } \\
\text { in Arbitrary } \\
\text { Units. }\end{array}$ \\
\hline 280 volts & 13.69 \\
\hline $420 \quad$ " & 14.90 \\
\hline $560 \quad$ " & 14.78 \\
\hline $700 \quad$ « & 14.74 \\
\hline
\end{tabular}

TABLE III. Pressure, 40 Atmospheres.

\begin{tabular}{c|c}
\hline Potential. & $\begin{array}{c}\text { Ionization } \\
\text { in Arbitrary } \\
\text { Units. }\end{array}$ \\
\hline 300 volts & 15.87 \\
600 " & 20.00 \\
900 " & 22.22 \\
1200 " & 22.27 \\
1500 " & 22.34 \\
\hline
\end{tabular}

It will be apparent from Table I. that for a voltage of 272 or more the current remained constant to within I per cent. The test for saturation voltage for the pressure of 20 atmospheres is given in Table II., which was obtained with the radium-bromide specimen in its position eight feet from the spheres. For the same position of the radium bromide the saturation voltage was also tested for a pressure of 40 atmospheres. The values obtained are given in Table III. It is evident from Tables II. and III., respectively, that 700 volts is amply sufficient for saturation 
at 20 atmospheres, and 1500 volts for 40 atmospheres when the $\mathrm{Ra} \mathrm{Br}$. specimen is in the position described.

\section{Main Measurements.}

General Procedure.-The method of making observations follows the same plan as that already indicated for the preliminary work. With the source of potential connected to the spheres as indicated in the diagram (Fig. I), and the fiber insulated from earth, the rate of deflection is proportional to the rate of production of ions. The general plan of making measurements will be apparent from the data given for a set of observations taken over the Mississippi river, May 16.

In order to avoid error due to leakage, the fiber is charged in such a way as to cross the zero during the observation and the range of scale divisions for any reading is so chosen that there is an equal number on each side

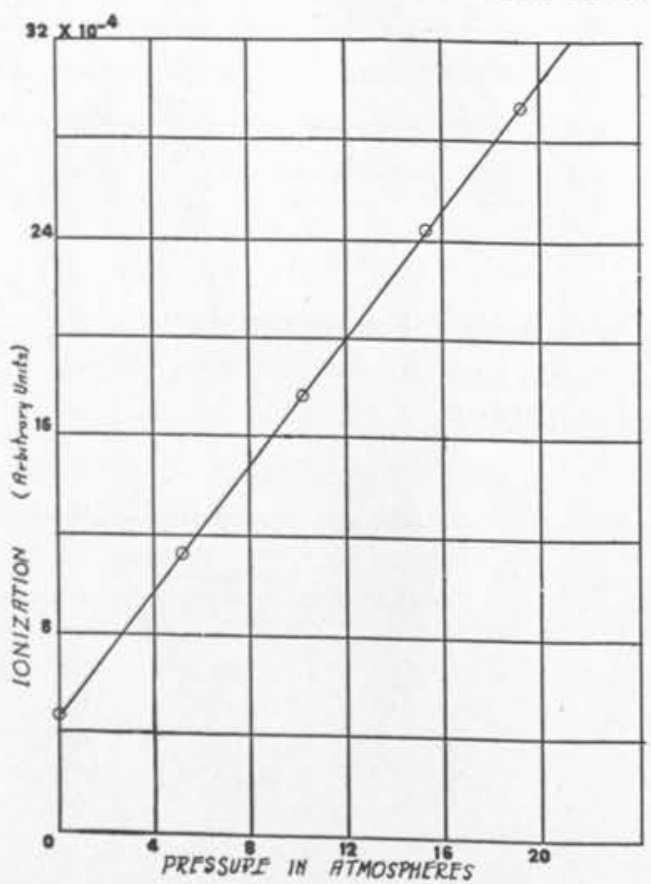

Fig. 3.

of the zero. As soon as the fiber passes the scale reading, $\theta_{1}$, the time $T_{1}$ is recorded. $\theta_{3}$ is the position of the fiber at the time $T_{3} . \Delta V$ is the change in the potential of the fiber in the time $T_{3}-T_{1}$. For the set of readings above, $\Delta V$ was always I volt. $S_{1}$, the sensitivity of the electrometer before the fiber was released, as well as $S_{2}$, the sensitivity after the fiber was earthed, were recorded. $P$ is the pressure in sphere 
$A$ in excess of one atmosphere. In order to test for constancy of conditions, the positions of the fiber were recorded both when the connection of the fiber to the calibrating device was released and when it was restored again at the end of the observation.

Curves Obtained.-The curve for the data of Table IV. is given in

TABLE IV.

\begin{tabular}{|c|c|c|c|c|c|c|c|c|c|}
\hline$\theta_{1}$. & $\theta_{3}$. & $T_{\mathrm{i}}$. & & r. & $\begin{array}{l}\Delta V \text { in } \\
\text { Volts. }\end{array}$ & $\begin{array}{c}\text { (Divisions per } \\
\text { Volt). }\end{array}$ & $\begin{array}{c}S_{2 .} \\
\text { (Divisions per } \\
\text { Volt). }\end{array}$ & $\begin{array}{c}\text { P. } \\
\text { (Atmos- } \\
\text { pheres). }\end{array}$ & $\begin{array}{c}\text { Time (Mid. } \\
\text { Pt. of } \\
\text { Reading. }\end{array}$ \\
\hline-4 & +4 & 0 & $\underset{5}{\operatorname{Min}}$ & $\begin{array}{r}\text { Sec. } \\
40.0\end{array}$ & 1 & 8 & 8 & 19.35 & 2:10 P.M. \\
\hline-4 & +4 & 0 & 6 & 47.6 & 1 & 8 & 8 & 15.35 & 3:00 P.M. \\
\hline-4 & +4 & 0 & 9 & 24.3 & 1 & 8 & 8 & 10.2 & 3:50 P.M. \\
\hline-4 & +4 & 0 & 14 & 43.0 & 1 & 8 & 8 & 5.12 & $4: 45$ P.M. \\
\hline-4 & +4 & 0 & 35 & 33.0 & 1 & 8 & 8 & 0. & 5:40 P.M. \\
\hline
\end{tabular}

Fig. 3. The reciprocals of the times (expressed in seconds) for the fiber to gain a potential of $\mathrm{I}$ volt are plotted against the pressure in $A$ in excess of one atmosphere. A linear relation was obtained. To find $q$,

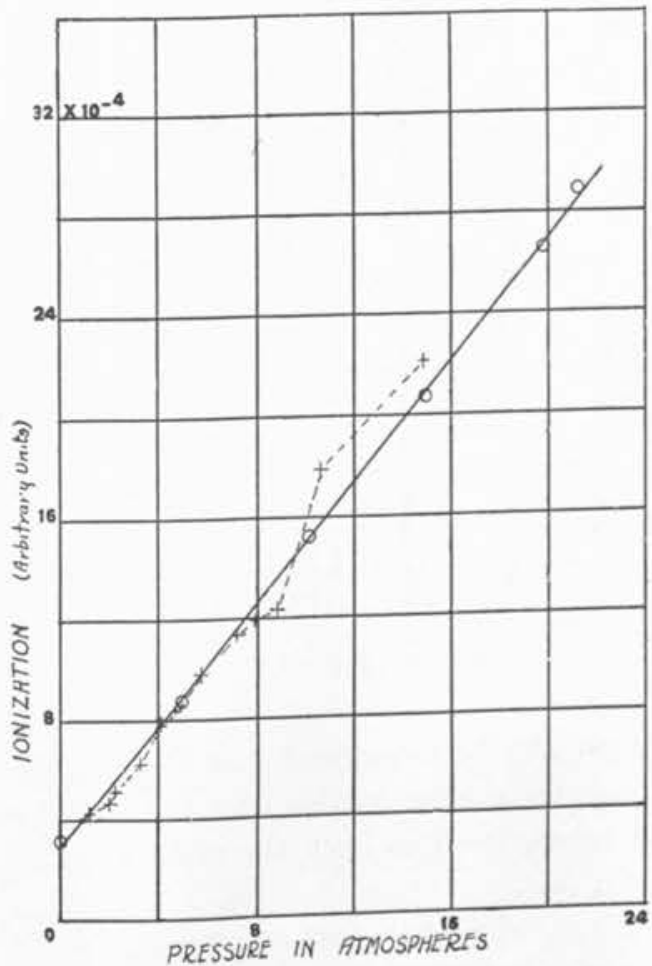

Fig. 4. 
the actual numbers of pairs of ions produced per c.c. per second, these ordinates are to be multiplied by $c(\mathrm{r} / 300) / v e$

$$
\begin{aligned}
(c & =\text { capacity of the system, } \\
\Delta V & =\mathrm{I} \text { volt }=\mathrm{I} / 300 \mathrm{e} . \mathrm{s} . \text { unit, } \\
v & =\text { volume of sphere } A \text { in c.c. } \\
e & =\text { electronic charge in e. s. units }) .
\end{aligned}
$$

The observations for the full line curve given in Fig. 4 were obtained over the river May 17 from 10:40 A.M. to 5:45 P.M.

The location of the apparatus over the water will be apparent from the photograph (Fig. 5). The water underneath the pier was $8 \frac{1}{2}$ feet

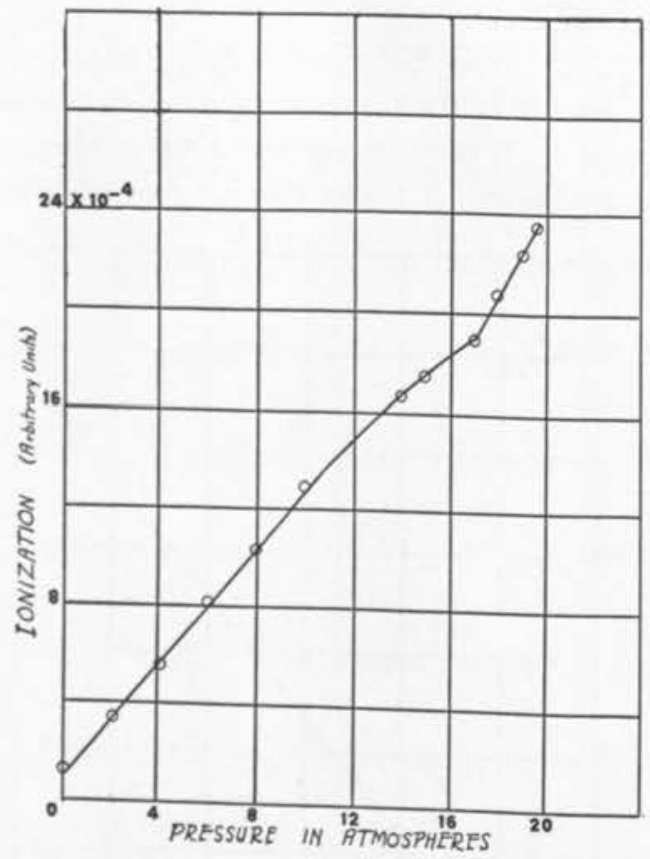

Fig. 6.

in depth. It might also be mentioned here that for the final measurements the electrometer was suspended from a rigid frame by means of springs so as to ensure freedom from the effect of any mechanical disturbances near the pier.

The curve in Fig. 6, which was obtained during the first week of May, is representative of other measurements taken in the laboratory. The 
Physte 11. Review, Vol. XVI., Second SERIES.

November, ro20.
Plate 1 .

To face page 430

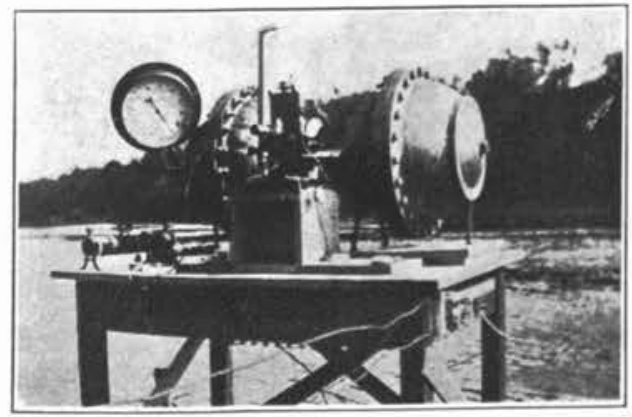

Fig. 5 .

K. MELVINA DOWNEY. 
measurements for Fig. 7 were made in the laboratory when radium was placed 8 feet from the spheres as previously indicated. The ordinates for Fig. 4, Fig. 6 and Fig. 7 are the same as have been given for Fig. 3 .

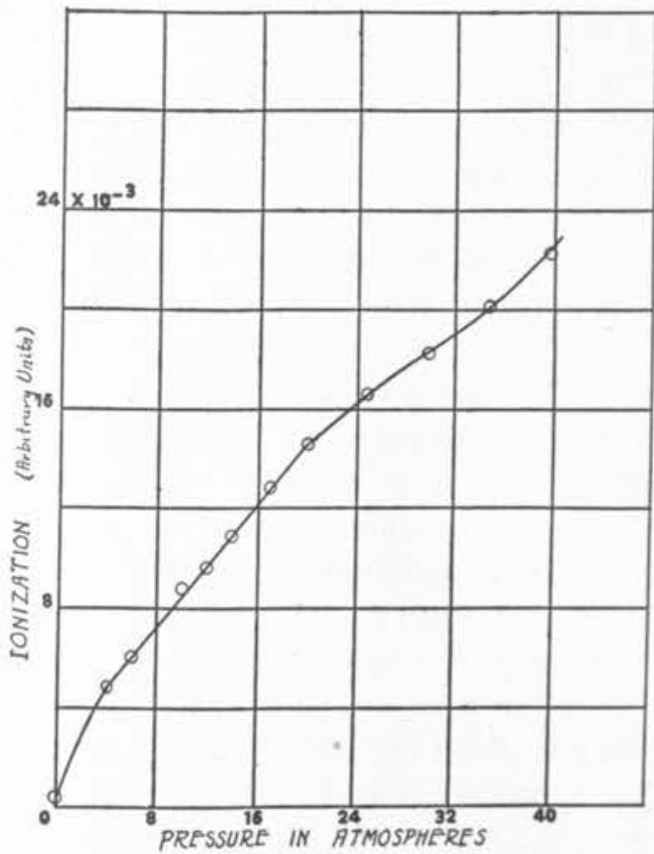

Fig. 7.

\section{Discussion.}

Ionization Pressure Relations.-If the residual ionization is due for the most part to radio-active impurities in the walls of the vessel, the radiation may be of the $\alpha, \beta$, or $\beta$ and $\gamma$ type, or it may be composed of all three types. The main part of this ionization would be due to the $\alpha$ and $\beta$ rays. The $\alpha$ rays which are the more efficient ionizers would be absorbed by about five $\mathrm{cm}$. of air at atmospheric pressure. While the $\beta$ rays and the secondary corpuscular rays due to them are more penetrating, practically all the soft $\beta$ rays and some of the hard rays, which have a range of several meters in air, would be absorbed at higher pressures. Thus, to the extent that the ionization is due to absorbable radiation of this kind, the ionization per gram of gas should decrease with increase of pressure. If one admits the existence of a penetrating radiation partaking of the $\gamma$ ray nature, the ionization in the closed vessel may be attributed to several sources: 
(I) The direct action of the penetrating radiation in the gas;

(2) The secondary radiation emitted from the gas by the primary penetrating radiation;

(3) The secondary radiation emitted from the walls of the vessel by the penetrating radiation.

In addition to these agencies it is of course possible that a portion of the ionization may be due to radio-active impurities. The portion of the ionization under (I) should be proportional to the pressure. Since the number of ions produced by the source (2) varies with the number of molecules of the gas, and with the number of electrons responsible for the ionization, it is apparent that this portion of the ionization would vary proportionally with the square of the pressure. This holds only to the extent that the ionization per $\mathrm{cm}$. of path of the electrons is the same at all parts of the path, and to the extent that the penetration of the electrons enables them to reach the walls before coming to rest. When the pressure is so high that the electrons complete their paths within the vessel, the increase of ionization would be less rapid than the square law would demand. But an influence in the opposite direction is to be found in the increase of ionization per $\mathrm{cm}$. of path toward the end of the range of the electrons. The latter effect would be apparent at pressures below that at which the former would appear. Consequently in regard to (2) it is possible that the curve of ionization against pressure might first show a variation according to the square of the pressure, then a more rapid increase than the square law would indicate, and finally a falling down to a linear relation when the pressure became so high that the penetration of all these ionizing electrons falls within the vessel.

The portion of ionization due to (3) should obey a linear law until the pressures become so high that the more active parts of the electrons' paths fall within the vessel. At such pressures the ionization would show a more rapid increase with pressure. Finally a saturation value should be reached when the whole path is completed within the vessel.

\section{Discussion of Experimental Results.}

A survey of Fig. 3 and Fig. 4 shows that the variation in the natural ionization with pressure obeys a linear law up to $2 \mathrm{I} .5$ atmospheres. In view of the preceding discussion, the linear relations given in Figs. 3, 4 and 5 indicate that the ionization within a closed vessel is not due to a soft radiation, but is determined either directly by a penetrating radiation of the $\gamma$ type or by a hard corpuscular radiation emitted from the walls of the vessel by the penetrating radiation. If it is attributed to 
the corpuscular radiation, this radiation itself is then so penetrating that it is not appreciably absorbed in passing through a $\mathrm{I}$-foot thickness of air at 20 atmospheres pressure. (One foot of air at 20 atmospheres pressure is equivalent in absorbing power to 20 feet of air at one atmosphere.)

It is further apparent that for the range of pressures employed, the contribution to the ionization by secondary rays emitted from the gas by the penetrating radiation is not the primary agency at work, for, if it were, the curve should become convex to the horizontal axis.

That moisture may produce an appreciable effect is shown by the curve of Fig. 6 which has a slight irregularity in the neighborhood of 17 atmospheres. This curve was obtained without any additional drying agent other than that in connection with the compressor of the liquid air machine. For the curves given in Figs. 3, 4 and 7 the drying agent was used as mentioned. For these no such irregularity was noticed. It is probable that there was some water vapor present in the case of (6). If the water vapor in the air from the compressor were as much as one seventeenth saturated, water would begin to condense at about 17 atmospheres and this would result in a change of conditions at this pressure. (Note: The sphere $A$ was filled with air at the highest pressure used and the air was allowed to escape very slowly to reduce to the lower pressures.) If one considers the actual numbers of ions given by Fig. 3 and Fig. 4 and compares them with the corresponding quantities for Fig. 6, it will be observed that there is little difference-those in the laboratory were slightly lower. Since the $8 \frac{1}{2} \mathrm{ft}$. of water was amply sufficient to cut out practically all the radiation from the soil below in the case of the measurements over the river, it seems evident that the concrete base of the building served the same function as the water. This was likewise indicated by the experiments of Miss Herrick, previously mentioned in this paper. For the work inside there was also the absorbing effect of the roof and the upper floors of the building.

It will be noted that the curves given in Figs. 3,4 and 6 show a noticeable ionization for zero pressure. This is no doubt due to a soft radiation from the walls of the sphere $A$, in which the pressure was varied. Before the compressed air was admitted into sphere $A$, it was left in a cylinder for a time so as to allow any radium emanation present to decay partially. However since the cylinder containing the air was not allowed to stand the same length of time before each set of observations, there would be different amounts of emanation admitted into the sphere. For the observations at the higher pressures, it was found necessary to wait a time after the pressure was lowered for a constant rate of deflection of 
the electrometer. This was due to the fact that it required time for the old decay products to come into equilibrium with the new amount of radium emanation. (A part of the emanation would be removed with the air while the pressure was being lowered.) It may be mentioned here that the radium emanation in air at ordinary atmospheric pressure is not responsible for more than I.7 ions per c.c. per second.

For the purpose of comparison it was naturally of interest to test the ionization produced due to the effect of $\gamma$ rays of radium when the pressure within the sphere $A$ was varied. What has been given in the discussion of the ionization pressure relations under (I), (2) and (3) may be considered as applying here-the penetrating radiation for the case being the $\gamma$ rays of radium and the secondary radiation from the walls and gas being rays of the $\beta$ and $\gamma$ type.

The curve of Fig. 7 indicates an increase of ionization with pressure. The ionization per gm. of air at pressures below 4 atmospheres is obviously somewhat larger on account of the soft radiation which is then effective. In curve 7 which has an approximately linear relation for the range of pressures from 4 to 20 atmospheres, it is noticed that the slope changes at about 20 atmospheres to a value approximately 0.6 of that for the pressures below 20 atmospheres. This departure from linearity is doubtless due to those $\beta$ rays which complete their paths within the spheres at this pressure. Twenty atmospheres would be sufficient to absorb $\beta$ rays having a penetration as great as 6 meters in air in one atmosphere. Those having a longer range would still be unabsorbed as would also those $\beta$ rays having their paths along the shorter chords of the sphere. The results at the highest pressure indicate a tendency toward a more rapid increase of the ionization with pressure. This suggests an effect due to the secondary radiation from the air. However, it is not possible to attach much importance to this until observations have been made for still higher pressures.

It is desirable to compare the results given in this investigation with these found by other observers. Patterson, who measured the natural ionization in a closed vessel for pressures extending only to $80 \mathrm{~cm}$ of mercury, obtained results which indicate a marked departure from linearity over this range. From this Patterson draws the conclusion that the natural ionization is due to radio-active impurity in the vessel. The actual ionization for one atmosphere was as much as 40 ions per c.c. per second, so that without question there was an unusually large amount of radio-active impurity in the walls of the vessel used by Patterson. A large number of the ions he obtained was probably due to the $\alpha$ particles so that he naturally found the effect per gram to diminish 
with increase of pressure (on account of the short range of the $\alpha$ particles). In reality the true residual ionization can have formed only a small part of the total effect measured in his experiment. In the writer's experiment results were never considered reliable unless the vessel had been so thoroughly cleaned that the ionization ranged from 10 to 13 or 14 ions per c.c. per second.

Additional results on natural ionization which were obtained by McLennan and Burton indicate an increase of ionization for the range of pressures used ( 4.4 to $500 \mathrm{c.m}$.), but the graph was not linear. The slope of the tangent of curve at one atmosphere is about twice that at seven atmospheres. Unfortunately McLennan and Burton have not stated any actual values for " q." It is therefore impossible to form any opinion as to whether the departure from linearity may have been due to radio-active impurities in the walls of the vessel. However, they do give some evidence of the existence of the radio-active impurities in their experiments.

If the ionization in a closed vessel is primarily due to the corpuscles emitted from the walls of the vessel by the external radiation, departure from linearity is to be expected when the pressure attains a value so high that the linear dimensions of the vessel become comparable with the electronic penetration at that pressure. Thus in comparing the results of the different observers it is to be noted that the departure from linearity is determined not by the pressure, but by the product of the pressure and a quantity of the order of the linear dimensions of the vessel. In other words, experiments in a chamber of average dimensions, $30 \mathrm{~cm}$., give at 20 atmospheres as much information in regard to this phase as experiments made up to 60 atmospheres in a vessel of only $10 \mathrm{~cm}$. linear dimensions.

The results of W. Wilson are of interest in that they indicate an increase of the natural ionization with pressure. While he used pressures as high as 45 atmospheres, the results he obtained can be compared with those of the writer only at those pressures below 15 atmospheres since his ionization chamber had less than $\frac{1}{3}$ the dimensions of that used in the present investigation. The electroscope which recorded the measurements consisted of a vessel ro $\mathrm{cm}$. cube. This was also used as the ionization chambers. The small sensitivity and large leakage in Wilson's experiments made it impossible for him to obtain results with a high degree of accuracy. The electroscope leaf was maintained at a potential of 200 volts. Since in the writer's experiments the change in potential of the fiber by the natural ionization was not over $I$ volt in the time necessary for a reading, it is evident that Wilson's leaf system could not 
have registered this with any high degree of accuracy. The writer has plotted in Fig. 4 the values of Wilson for pressures above one atmosphere (these are indicated by means of crosses on the dotted curve). The slope of the linear part of Wilson's curve has been made the same as that of the line obtained by the writer. It will be noted that the departure from linearity of the author's results is never more than 1.30 per cent. of the highest value measured while the corresponding quantity for Wilson's results is as high as 10.50 per cent.

Wilson also obtained an ionization pressure curve for the case where radium-bromide was placed outside the ionization chamber and the pressures were varied up to 40 atmospheres. From what has been said it is clear that this would amount to a range of approximately $\mathrm{I} 3$ atmospheres in the writer's experiments. Wilson obtains a maximum at this pressure probably for the reason that he did not have saturation voltage. At atmospheric pressure he works with a value for $q$ amounting to 216 ions per c.c. per second which is almost four times that obtained in the present experiment at atmospheric pressure. It was possible for the writer to work with a weak radio-active specimen on account of the compensating arrangement (the 2 spheres in combination with the ten megohm resistance). Furthermore the radium-bromide was shielded to such an extent that the $\gamma$ rays reaching the ionizaiton chamber and consequently the secondary corpuscular radiation due to the $\gamma$ rays were much harder than the radiation which was responsible for the ionization in Wilson's experiments. This would naturally cause Wilson's curve to depart from linearity sooner than the writer's curve.

Additional measurements on the ionization in a closed vessel due to the effect of the $\gamma$ rays of radium have been made by Kaye and Laby ${ }^{1}$ as well as D. C. H. Florance, ${ }^{2}$ but it will be seen that the range of pressures is rather limited when compared with the conditions of the present investigation. These observers worked with ionization vessels the plates of which were not over $2 \mathrm{~cm}$. apart. In the work of Kaye and Laby there was a central electrode about $\frac{1}{2} \mathrm{~cm}$. from the sides of the vessel, so that as regards ionization by electrons emitted from the walls of the vessel their curve, which was obtained for a range of pressures up to 17 atmospheres, could be compared with that of the writer only for pressurse below one atmosphere.

D. C. H. Florance who used pressures up to 80 atmospheres worked with the plates $\mathrm{I} \mathrm{cm}$, apart, with an additional reading for a distance of $2 \mathrm{~cm}$. between the plates. For the spheres used in the present experi-

${ }^{1}$ Kaye and Laby, Phil. Mag., Ser. 6, Vol. 16, p. 879, 1908.

2D. C. H. Florance, Phil. Mag., Ser. 6, Vol. 25, p. 172, 1913. 
ment, the former condition would correspond to a pressure of $\mathbf{5}$ atmospheres and the latter to a pressure of ro atmospheres. The inability of Florance to secure saturation voltage at the higher pressures is no doubt due to the unusually large amount ( $30 \mathrm{mg}$.) of radium-bromide used. Without saturation a large change in slope would be expected at the higher pressures.

In conclusion it is a pleasure to acknowledge my gratitude to Professor W. F. G. Swann, who has suggested the problem and directed the investigation. To him I extend my best thanks for the encouragement and advice has has given throughout this work.

I wish to thank Professor H. A. Erikson for his continued interest in the work, and also Mr. C. H. Dane, mechanician, for assistance in the solution of mechanical difficulties.

DEPARTMENT OF PHysics,

UNIVERSITY OF MINNESOTA,

December, I9I9. 